\title{
Evaluation De La Diversité Et Estimation De La Biomasse Aérienne Des Arbres Du Jardin Botanique De Bingerville (District d'Abidjan, Côte d'Ivoire)
}

\author{
Monssou Eugène Oulaïtar, Doctorant \\ Vroh Bi Tra Aimé, Assistant \\ Laboratoire de Botanique, UFR Biosciences, \\ Université Félix Houphouët-Boigny, Abidjan, Côte d’Ivoire
}

Goné Bi Zoro Bertin, Maître-assistant Adou Yao Constant Yves, Maître de Conférences

Laboratoire de Botanique, UFR Biosciences, Université Félix HouphouëtBoigny, Abidjan, Côte d'Ivoire ; Département Recherche et Développement,

Centre Suisse de Recherches en Côte d'Ivoire (CSRS)

N'Guessan Kouakou Edouard, Professeur Titulaire

Laboratoire de Botanique, UFR Biosciences,

Université Félix Houphouët-Boigny, Abidjan, Côte d’Ivoire

doi: 10.19044/esj.2016.v12n6p168 URL:http://dx.doi.org/10.19044/esj.2016.v12n6p168

\begin{abstract}
The Bingerville Botanical Garden is an urban green space which can contribute to the quality of the living environment and to attractivity of tourists in Abidjan city. Its floristic diversity and socio-economic and ecological benefits remain still unknown. The present study was conducted to assess botanical species diversity and the reduction of $\mathrm{CO}_{2}$ emissions in this space. Trees species with $\mathrm{dbh} \geq 10 \mathrm{~cm}$ were recorded in 41 plots of 500 $\mathrm{m}^{2}$ surveyed in four types of biotopes: two arboretums with 20 and 100 years old, one secondary forest with more than 100 years and a fallow of 20 years old. Floristic indices and aboveground biomass (AGB) were determined for each biotope. A total of 742 trees with $\mathrm{dbh} \geq 10$ were counted: 127 species. Preponderance species are Cassia siamea, Terminalia mantaly, Milicia excelsa and Ceiba pentandra in arboretums, Pentadesma butyracea and Acacia mangium in the secondary forest, Ficus exasperata and Borassus aethiopum in the fallow. Arboretums are richest (96 species), and they have more AGB (566.7 t/ha). The secondary forest has 71 species with an aboveground equal to $381.4 \mathrm{t} / \mathrm{ha}$. The fallow is less rich (13 species) with an AGB of $21 \mathrm{t} / \mathrm{ha}$. This study has shown the importance of Bingerville Botanical Garden in plant and fighting against climate change.
\end{abstract}


Keywords: Aboveground biomass, ecosystem services, climate change, biodiversity, Ivory Coast

\section{Résumé}

Le Jardin Botanique de Bingerville (JBB) est un espace vert urbain qui peut contribuer à la qualité du cadre de vie et à l'attractivité des touristes dans la ville d'Abidjan. Ses caractéristiques floristiques et ses bienfaits socio-économiques et écologiques demeurent encore mal connus. La présente étude a été menée pour mettre en exergue le rôle que peut jouer cet espace dans la conservation des espèces végétales et la réduction des émissions de $\mathrm{CO}_{2}$. Des inventaires d'espèces arborescentes de $\mathrm{dbh} \geq 10 \mathrm{~cm}$, ont été réalisés dans 41 placettes de $500 \mathrm{~m}^{2}$ mises en place dans quatre types de biotopes: deux arboretums âgés de 20 et 100 ans, une forêt secondaire de plus de 100 ans et une jachère de 20 ans. Des indices de diversité et la composition floristiques ainsi que la biomasse végétale aérienne ont été déterminés pour chacun de ces biotopes. Au total 742 arbres de $\mathrm{dbh} \geq 10 \mathrm{~cm}$ ont été recensés. Ils se répartissent entre 127 espèces dont les plus prépondérantes sont Cassia siamea, Terminalia mantaly, Milicia excelsa et Ceiba pentandra dans les arboretums, Pentadesma butyracea et Acacia mangium dans la forêt secondaire, Ficus exasperata et Borassus aethiopum dans la jachère. Les arboretums sont les plus riches (96 espèces); ils présentent la plus forte biomasse aérienne (566,7 t/ha). La forêt secondaire avec 71 espèces, a une biomasse de 381,4 t/ha. La jachère a la plus faible richesse (13 espèces) avec une biomasse aérienne de $21 \mathrm{t} / \mathrm{ha}$. Cette étude démontre l'importance du Jardin Botanique de Bingerville dans la conservation de la diversité arborée et la séquestration du carbone atmosphérique.

Mots-clés: Biomasse végétale, services écosystémiques, changement climatique, biodiversité, Côte d’Ivoire

\section{Introduction}

La destruction des forêts s'est particulièrement accentuée en zone tropicale au cours des 50 dernières années pendant lesquelles l'être humain a modifié l'environnement plus rapidement et dans des proportions plus vastes qu'à toute autre période comparable de l'histoire de l'humanité (FAO, 2011). Cette destruction engendre une perte de la diversité biologique. Il parait donc urgent de prendre des mesures allant dans le sens de la sauvegarde de ce qui reste des forêts tropicales afin de conserver la biodiversité.

L'état de Côte d'Ivoire, dans le souci de faire face aux problèmes de déforestation, a mis sur pied des structures telles que la Société de 
Développement des Forêts (SODEFOR) pour les forêts classées et l’Office Ivoirien des Parcs et Réserves (OIPR), pour les parcs nationaux et réserves. Une des politiques de ces structures a été la création des parcs, réserves et jardins dans le milieu urbain. Il s’agit notamment du Parc National du Banco qui est l'un des plus connus. Dans ces territoires urbains, les forêts peuvent avoir des fonctions multiples et répondre à la demande des usagers qui ont d'importants besoins récréatifs et de ressourcement pour se maintenir en forme et compenser le stress de la vie moderne. Malheureusement, les populations riveraines, à la recherche d'espaces urbanisables et de terres cultivables ainsi que d'espèces à diverses vertus, exercent de fortes pressions sur ces forêts urbaines si bien que leurs surfaces ne cessent d'être grignotées. Pourtant, ces écosystèmes forestiers urbains fournissent de nombreux services environnementaux dont l'un des plus importants et nécessaires au maintien de la vie, est la séquestration de carbone atmosphérique (Nowak et al., 2006). En effet, le bon fonctionnement de l'environnement urbain dépend de la diversité de vie présente dans les parcs urbains (Vroh et al., 2014). L'état critique que présente la biodiversité dans ces milieux, emmène à prendre des mesures allant dans le sens de la sauvegarde de ce qui reste des forêts urbaines.

Dans la perspective de protection de la biodiversité urbaine, l'avenir des ressources forestières et arborées urbaines est désormais au cœur de plusieurs débats environnementaux ou de développement organisés par les ministères en charge de l'environnement en Côte d'Ivoire. Ces réflexions organisées pour avoir des villes plus durables appellent à un développement des surfaces vertes dont la gestion doit être inscrite dans un programme de foresterie urbaine. Dans la ville d'Abidjan, des études sur cette thématique de la diversité et estimation du stock de carbone, ont été réalisées au Parc National du Banco (N’Guessan et al., 2015), dans les jardins de la Commune du Plateau (Vroh et al., 2014) et au Centre National de Floristique (Kouamé, 2013). Jusqu’à présent, aucune étude sur la biomasse des arbres n’a été abordée dans le Jardin Botanique de Bingerville (JBB). Parmi les travaux réalisés dans le JBB, figurent une étude sur le savoir-faire des populations locales des taxons de plante (Séguéna et al., 2013). Récemment, ce jardin a subi la perte de plus du quart de sa superficie au profit de l'urbanisation. Il a été aussi l'objet d'exploitation agricole dans les zones périphériques. Laissé dans un état d'abandon pendant plusieurs années, il fut réhabilité en 1996. Son administration est actuellement assurée par les services des eaux et forêts qui ambitionnent y mener une gestion durable. Cette gestion nécessite la connaissance de son état scientifique et des services qu'il procure aux populations. La présente étude a été réalisée pour mettre en relief le rôle et l'importance de ce jardin, ouvert aux usages récréatifs. Plus spécifiquement, l'étude a consisté à déterminer la valeur de la diversité arborée du JBB et à 
estimer la biomasse afin d'en déduire la quantité de carbone pouvant être stockée dans les arbres.

\section{Méthodes d'étude Site d'étude}

La ville de Bingerville anciennement située à une dizaine de kilomètres d'Abidjan, se trouve aujourd'hui, avec l'extension de celle-ci, à moins de $2 \mathrm{~km}$. La végétation originelle dont la plus grande partie a été détruite dans le cadre de l'extension de la ville d'Abidjan, était caractérisée par des forêts denses sempervirentes à Turraeanthus africanus et Heisteria parvifolia (Mangenot, 1955). Le climat correspondant est de type Subéquatorial (Edlin, 1971) caractérisé par des températures de faibles amplitudes de $24^{\circ} \mathrm{C}$ à $28^{\circ} \mathrm{C}$ et des précipitations abondantes, qui atteignent 1547 mm (Kouassi, 2010).

Le Jardin Botanique de Bingerville (Figure 1), objet de cette étude, a été créé par l'administration coloniale en conformité avec sa tradition qui voulait qu'à chaque proximité de poste militaire ou administratif, un jardin botanique soit mis en place. Précédemment Jardin d’Essai de Bingerville, sur décision du gouverneur par l’arrêté $N^{\circ}$ 150/04 du 17 Février 1904, il devint Jardin Botanique de Bingerville. Ce Jardin est parcouru de voies internes délimitant plusieurs types d'espaces. Dans le cadre de cette étude, quatre de ces espaces ont été étudiés, il s’agit de deux arboretums dont l'un âgé de plus de 100 ans est issu de l'extension progressive du jardin depuis sa création par l'administration coloniale en 1904, et l'autre âgé de 20 ans qui résulte d'un ensemble de parcelles octroyées à diverses structures (Organisation des Nations Unies en Côte d'Ivoire, J'aime Israël, Standard Chartered Bank...) dans le cadre d’activités de reboisement depuis 1996. Il s'agit d'une forêt ancienne de 4 ha, âgée de plus de 100 ans dont la présence remonte à la création du jardin botanique et une jachère de 20 ans issue de champs de vivriers réalisés par les riverains pendant la période d'abandon du jardin.

\section{Méthode de collecte de données}

L'inventaire a été conduit à l'aide de la méthode des relevés de surface. Au total, 41 relevés ont été effectués dans des placettes de $500 \mathrm{~m}^{2}$ dans les quatre biotopes à raison de 10 pour l'arboretum de plus de 100 ans, la forêt, et la jachère et 11 pour l'arboretum de 20 ans. Les mesures n'ont pris en compte que les arbres de $\mathrm{dbh} \geq 10 \mathrm{~cm}$ car selon Weldenson, (2010), ceux-ci sont les éléments dominants de la biomasse aérienne dans les forêts tropicales. 
L'identification des espèces a été faite par comparaison des spécimens récoltés à ceux de l’herbier du Centre National de floristique de l’Université Félix Houphouët Boigny.

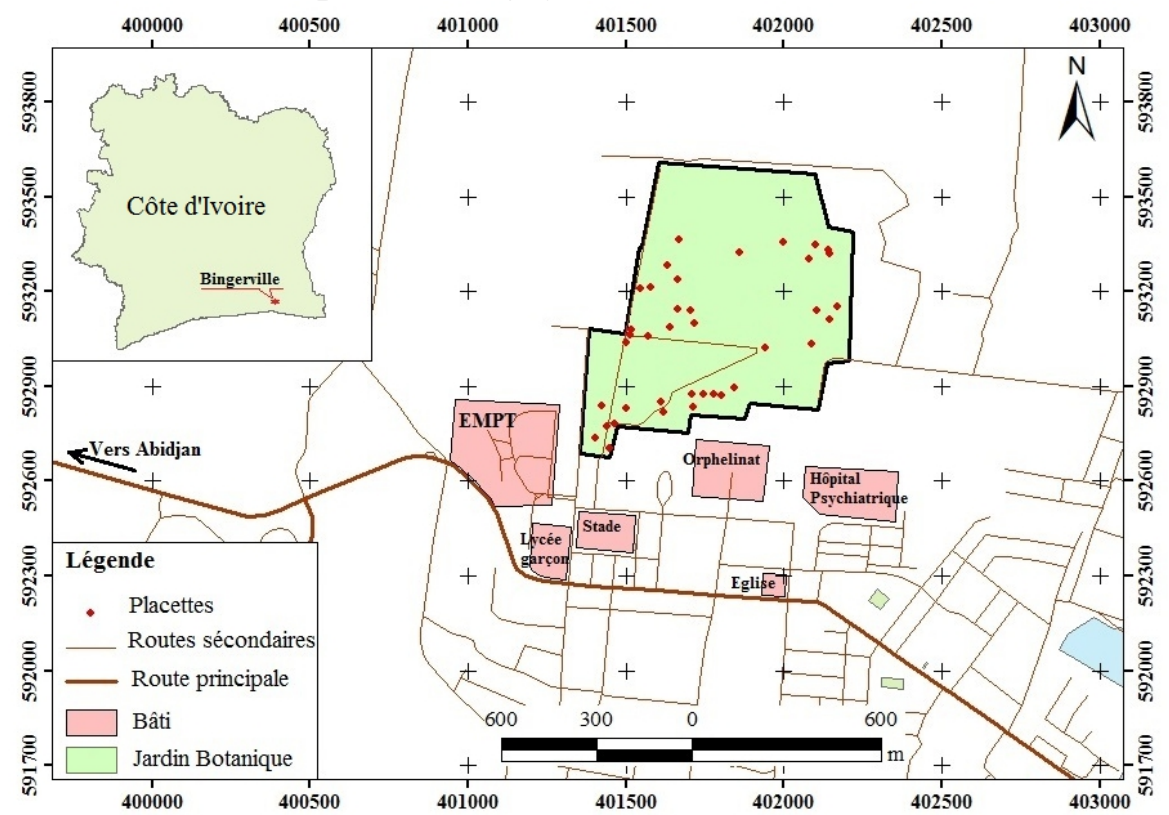

Figure 1 : Carte de localisation du JBB et des placettes d'inventaire

\section{Analyse des données}

La richesse, la composition et la diversité au niveau de la flore, ont été déterminées. Il s'est agit d'abord pour chacune des espèces présentes dans les différents biotopes, d'identifier le genre, la famille et les types chorologiques et biologiques (Raunkier, 1934 et Aké-Assi, 2001-2002). La richesse spécifique a été obtenue par addition du nombre total d'espèces arborées recensées par type de biotope. Il a été également dénombré les espèces à statut particulier dans chaque biotope. Il s'agit des espèces endémiques de la Haute Guinée, Ouest africaines et ivoiriennes, des espèces inscrites sur la liste rouge UICN (2015) et des espèces devenues rares et menacées d'extinction selon Aké-Assi (1998). Ensuite, les indices de diversité de Shannon (1948) et d'équitabilité de Piélou (1966) ont permis d'estimer la diversité des espèces arborescentes dans chaque type d'espace. L'indice de Shannon a permis d'apprécier la diversité des espèces des différents types de biotopes. Il mesure la composition en espèces d'un peuplement en combinant l'abondance relative des espèces et la richesse spécifique. Il se résume à la formule suivante :

$$
\mathrm{H}^{\prime}=-\sum \mathrm{pi} \times \ln \mathrm{pi}
$$

Dans cette formule, pi est la proportion d'une espèce i par rapport au nombre total d'espèces. 
L’équitabilité (J’) a renseigné sur la répartition des arbres entre les différentes espèces d'un peuplement. Sa formule est la suivante :

$$
\mathrm{J}^{\prime}=\mathrm{H}^{\prime} / \ln \mathrm{S}
$$

Avec $H^{\prime}$ la diversité de Shannon et $\mathrm{S}$ la richesse spécifique.

Le coefficient de similitude de Sørensen (1948) a servi pour exprimer le degré de ressemblance spécifique entre les espaces en les comparant deux à deux. Plus les deux listes floristiques comparées ont des espèces en commun, plus leur coefficient tend vers 100 .

Pour apprécier l'importance de chaque espèce au sein des différents espaces, nous avons utilisé l'Indice de Valeur d'Importance (IVI) de Cottam et Curtis (1956). L'IVI donne pour chacune des espèces des informations sur le nombre d'individus, sur leurs distributions ainsi que sur leur importance en fonction de la surface terrière (Nusbaumer et al., 2005). Il se calcule de la manière suivante:

$$
\mathrm{IVI}=\mathrm{FR}+\mathrm{D}_{\mathrm{o}} \mathrm{R}+\mathrm{D}_{\mathrm{e}} \mathrm{R}
$$

Où $F R, D_{0} R$ et $D_{e} R$ désignent respectivement la fréquence relative, la dominance relative et la densité relative de chaque espèce.

Au niveau de la structure de la végétation de chaque espace, la densité des arbres et l'aire basale totale ont été déterminées. Elles se définissent respectivement par le nombre d'individus par unité de surface pour la densité et la superficie occupée par le tronc, mesuré sur l'écorce à 1,30 m du sol, pour l'aire basale. Les arbres ont été répartis par classe de diamètre et les histogrammes de distribution des tiges ont été dressés pour caractériser les structures diamétriques des végétations.

Aussi, les biomasses aériennes des différents individus ont-elles été déterminées à l'aide du modèle standard de régression de Chave et al (2005) pour les forêts tropicales humides. Cette équation se définie comme suit :

$$
\mathrm{Ba}=\rho \cdot \exp \left[-1,499+2,148 \ln (\mathrm{dbh})+0,207 \ln (\mathrm{dbh})^{2}-0,0281 \ln (\mathrm{dbh})^{3}\right]
$$

Dans cette formule, Ba est la biomasse aérienne exprimée en kg et dbh, le diamètre mesuré sur le tronc à $130 \mathrm{~cm} \mathrm{du} \mathrm{sol;} \rho$ est la densité spécifique d'une espèce exprimée en $\mathrm{g} / \mathrm{cm}^{3}$. Pour les espèces dont nous ne connaissions pas la densité, nous avons utilisé la valeur par défaut ( $\rho_{\text {défaut }}=$ $0,58 \mathrm{~g} / \mathrm{cm}^{3}$ ) pour les forêts tropicales d'Afrique.

Lorsque le dbh est supérieur à $156 \mathrm{~cm}$, l’équation appliquée pour le calcul de la biomasse est celle de Chave et al. (2004) pour la zone Pantropicale :

$$
\mathrm{Ba}=(\rho / 0,6) \exp \left[-3,742+3,450 \ln (\mathrm{dbh})-0,148 \ln (\mathrm{dbh})^{2}\right]
$$

Ces équations de Chave et al. (2004; 2005) ne sont valables pour l'estimation de la biomasse des palmiers (palmier à huile, cocotiers et rôniers). L'équation utilisée pour la biomasse des palmiers (palmier à huile, rônier et cocotier) est celle développée par Brown (1997) pour les forêts tropicales humides : 


$$
\mathrm{Ba}=\exp (-2,134+2,53 \ln (\mathrm{dbh}))
$$

La biomasse a été convertie en stock de carbone correspondant en multipliant par 0,5 (Brown et Lugo, 1992). Et la masse de dioxyde de carbone $\left(\mathrm{CO}_{2}\right)$ dans l'atmosphère est obtenue en multipliant celle du carbone par 0,27 (l'équivalent carbone est la mesure officielle des émissions de gaz à effet de serre).

Les valeurs moyennes de quelques descripteurs floristiques des différents biotopes ont été comparées à l'aide d'une Analyse de Variance à un facteur après vérification de la normalité des distributions et de l’homogénéité des variances. Les tests de significativité ont été réalisés grâce au test de Turkey à 5\%. Le logiciel $\mathrm{R}$ a été utilisé pour réaliser ces tests.

\section{Résultats}

\section{Richesse, Compositions et Diversité floristiques}

L'inventaire a permis de recenser 742 individus de $\mathrm{dbh} \geq 10 \mathrm{~cm}$ repartis entre 127 espèces, 101 genres et 39 familles. Le nombre d'espèces varie de 13 à 75 dans les biotopes (Tableau 1), pour une valeur moyenne de $53,25 \pm 28,33$. Le nombre d'espèces dans les placettes de l'arboretum de plus de 100 ans varie de 18 à 29, soit une valeur moyenne de 21,5 $\pm 3,97$. Le nombre d'espèces dans les placettes de la forêt varie de 18 à 28 soit une valeur moyenne de 23 $\pm 3,71$. Le nombre d'espèces dans les placettes réalisées dans la jachère varie de 2 à 13 ce qui correspond à une valeur moyenne de 5,5 $\pm 4,14$ et celui des placettes de l'arboretum de 20 ans varie de 18 à 30 , soit une valeur moyenne de $23 \pm 3,71$. Les espèces les plus importantes ayant au moins 10 p.c. des IVI totaux sont variables d'un espace à l'autre. Dans l'arboretum de plus de 20 ans, Cassia siamea est la première des espèces avec 110,6 p.c. des IVI, elle est suivie de Terminalia mantaly avec un IVI de 101,6 p.c. Dans la forêt secondaire, Pentadesma butyracea enregistre la plus forte valeur des IVI avec 331,41 p.c. Elle est suivie d'Acacia mangium qui a 127,42 p.c. des IVI. Pour la jachère, ce sont Ficus exasperata, Borassus aethiopum, Dialium dinklagei et Adansonia digitata, avec des valeurs respectives d'IVI de 41,03 p.c., 39,05 p.c., 31,22 p.c. et 31,12 p.c. qui sont les espèces les plus importantes. Quant à l'arboretum de plus de 100 ans, les espèces Milicia excelsa (140,96 p.c.) et Ceiba pentandra (IVI $=108,13$ p.c.) restent les plus importantes.

Les indices de Shannon varient d'un biotope à l'autre. L'arboretum de 20 ans et la forêt sont les plus diversifiés avec des valeurs moyennes respectives d'indice de 3,8 et 3,6. A l'opposé, la jachère reste le biotope le moins diversifié avec un indice de 2,3 (Tableau 1). La différence entre les valeurs moyennes de ces indices est significative $(F=38,06 ; p<0,0001)$. L'indice d'équitabilité suit la même tendance que celui de Shannon. Il varie de 0,9 en jachère à 0,8 dans les autres types de biotope (Tableau 1 ). 
Tableau 1: Récapitulatif de la richesse et de la diversité floristique des espaces inventoriés

\begin{tabular}{cccccc}
\hline Paramètres floristiques & Arboretum plus de 100 ans & Forêt & Jachèrt & Arboretum de 20 ans & Tota \\
\hline Espèces recensées & $54 \mathrm{~b}$ & $71 \mathrm{~b}$ & $13 \mathrm{a}$ & $75 \mathrm{~b}$ & 127 \\
Shannon & $3,2 \mathrm{~b}$ & $3,6 \mathrm{a}$ & $2,3 \mathrm{c}$ & $3,8 \mathrm{a}$ & 4,2 \\
Equitabilité & $0,8 \mathrm{~b}$ & $0,8 \mathrm{~b}$ & $0,9 \mathrm{a}$ & $0,8 \mathrm{~b}$ & 0,8 \\
Genres & 47 & 59 & 11 & 63 & 101 \\
Familles & 27 & 25 & 9 & 30 & 39 \\
Espèces exotiques (p.c.) & 48,1 & 25,4 & 38,5 & 36 & 36,2 \\
Espèces GC (p.c.) & 38,9 & 53,5 & 23 & 41,3 & 43,3 \\
Espèces GC-SZ (p.c.) & 13 & 19,7 & 30,8 & 20 & 18,9 \\
Espèces SZ (p.c.) & 0 & 1,4 & 7,7 & 2,7 & 1,6 \\
Mégaphanérophytes (p.c.) & 24,1 & 22,5 & 20,5 & 14,7 & 22 \\
Mésophanérophytes (p.c.) & 33,3 & 38 & 30,8 & 37,3 & 34 \\
Microphanérophytes (p.c.) & 42,6 & 39,5 & 61,5 & 48 & 44 \\
\hline
\end{tabular}

NB : Pour une même ligne, les valeurs affectées de lettres différentes sur une même ligne sont statistiquement différentes au seuil $5 \%$

Au total 36,2 p.c. d'espèces exotiques ont été inventoriées contre 63,8 p.c. d'espèces locales. Au niveau des types biologiques, les Mésophanérophytes et les Microphanérophytes sont les plus nombreuses dans l'ensemble des biotopes.

Les genres et les familles les plus représentés par type d'espace se présentent comme suit, pour l'arboretum de 20 ans, le genre Cassia (3 espèces) et la famille des Moraceae ( 7 espèces). Dans la forêt secondaire, le genre Cassia (4 espèces) et les familles des Sterculiaceae et Moraceae (7 espèces). Pour la jachère, il s'agit du genre Cassia (3 espèces) et la famille des Caesalpiniaceae (5 espèces). L'arboretum de plus de 100 ans est plus riche en genre Terminalia (4 espèces) et la famille des Euphorbiaceae (8 espèces).

Les coefficients de similitude de Sørensen calculés pour les espaces du JBB, montre qu'il y a dissemblance floristique entre les différents biotopes du JBB (Tableau 2).

Il a été dénombré 22 espèces végétales à statut particulier (Tableau 3) dont 19 appartiennent à la liste rouge de l'UICN (2015). Elles se répartissent en 77,3 p.c. d'espèces vulnérables, 9 p.c. d'espèces en danger. On note une espèce endémique à la Côte d'Ivoire (GCi) : Leptoderris miegei et trois endémiques au bloc forestier ouest africain (GCW) qui sont Berlinia occidentalis, Milicia regia et Tarrietia utilis. Cinq de ces espèces à statut particulier ne se rencontrent que dans les forêts de la région phytogéographique de Haute Guinée (HG) : Berlina occidentalis, Guarea cedrata, Leptoderris miegei, Milicia regia etTarrietia utilis. 
Tableau 2: Coefficient de similitude de Sorensen entre les différents biotopes du JBB

\begin{tabular}{ccccc}
\hline Biotopes & Arboretum de plus de 100 an: & Forêl Jachèr Arboretum de 20 an: \\
\hline Arboretum de plus de 100 an: & - & & & \\
Forêt & 35 & - & & \\
Jachère & 17,9 & 17,7 & - & \\
Arboretum de 20 ans & 40,3 & 42,6 & 22,7 & - \\
\hline
\end{tabular}

\section{Structure et biomasse du peuplement des biotopes}

La densité des tiges de $\mathrm{dbh} \geq 10 \mathrm{~cm}$ varie de 88 arbres/ha pour la jachère à 460 arbres/ha pour la forêt (Tableau 4). Les aires basales correspondantes sont de $1,04 \mathrm{~m}^{2} /$ ha et $14,59 \mathrm{~m}^{2} /$ ha respectivement pour la jachère et la forêt. L’ensemble des espaces inventoriés donne un total de 742 individus ayant au moins un diamètre à hauteur de poitrine de $10 \mathrm{~cm}$, ce qui correspond à 362 arbres/ha pour une aire basale totale de 39,72 m²/ha.

Les valeurs de la biomasse par espace varient de 21 t/ha pour la jachère à 381,4 t/ha pour la forêt secondaire (Tableau 4). Pour tout le jardin botanique, le stock individuel de carbone est de 0,6 t/ha/tige. Ce stock de carbone par tige varie quand on considère les types d'espaces, il est plus élevé dans la forêt avec 0,8 t/ha/tige, suivie par les deux arboretums avec 0,6 t/ha/tige et la jachère 0,2 t/ha/tige. Les variations des valeurs d'équivalent $\mathrm{CO}_{2}$ suivent les mêmes tendances observées pour les stocks de carbone, dans les différents espaces.

La distribution des tiges dans les classes de diamètre montre que lorsqu'on passe des petites classes aux grandes, le nombre de tiges diminue dans les différents espaces, sauf celui de l'arboretum de plus de 100 ans. Ceci se traduit par un histogramme de distribution en forme de demi cloche pour l'arboretum de plus de 100 ans et des histogrammes en « J inversé » pour les autres espaces (Figure 2).

\section{Discussion}

Les parcelles du JBB ont des richesses spécifiques différentes les unes des autres. Ainsi, la jachère reste moins riche en espèces arborées par rapport aux autres parcelles. Cela pourrait s'expliquer par la pratique de l'agriculture qui a dégradé les conditions favorables à l'installation de la majorité des espèces arborescentes rencontrées dans les autres parcelles. Des observations de terrain ont permis de constater qu'elle est plus riche en espèces herbacées qui sont pour la majorité, des espèces pionnières (Bakayoko, 2005). L'arboretum de 20 ans est le plus riche en espèces arborées (75 espèces). Cette grande richesse observée est due au fait que plusieurs espèces ont été plantées pendant la mise en place de l’arboretum. Cette diversité continue de s'accroitre à chaque campagne de reboisement lancée dans le JBB. Aussi, les effets du nettoyage dans l'arboretum de 20 ans sont-ils moins perçus comparativement à celui de l'arboretum de plus de 100 
ans, où cette activité d'entretien est très visible. La forêt secondaire est riche et diversifiée. Âgée de plus de 100 ans, ce biotope favorise le retour d'un certain nombre d'espèces arborescentes. Cette observation confirme l'idée selon laquelle la richesse d'un milieu augmente avec le temps (Puig, 2001).

Tableau 3: Liste des espèces à statut particulier, recensées dans les biotopes inventoriés dans le Jardin Botanique de Bingerville

\begin{tabular}{|c|c|c|c|c|c|c|c|c|}
\hline \multirow{3}{*}{ espèces } & \multirow{3}{*}{ Familles } & \multicolumn{3}{|c|}{ statuts particulier } & \multicolumn{4}{|c|}{ Biotopes } \\
\hline & & & & & Arboretun & & & Arboretun \\
\hline & & Endér & misn & UICN & $\begin{array}{c}\text { de plus de } \\
100 \text { ans }\end{array}$ & & |chèr & $\begin{array}{c}\text { de plus de } \\
20 \text { ans }\end{array}$ \\
\hline Afzeliaafricana & Caesalpiniaceaı & - & - & $\mathrm{Vu}$ & - & + & - & - \\
\hline Berliniaoccidentalis & Caesalpiniaceaı & HG & GCh & - & - & + & - & - \\
\hline Cola reticulata & Sterculiaceae & - & - & $\mathrm{Vu}$ & - & + & - & - \\
\hline Entandrophragmaangolense & Meliaceae & - & - & $\mathrm{Vu}$ & - & + & - & + \\
\hline Entandrophragmacylindricun & Meliaceae & - & - & $\mathrm{Vu}$ & - & + & - & + \\
\hline Entandrophragma utile & Meliaceae & - & - & $\mathrm{Vu}$ & - & - & - & + \\
\hline Erythrinavogelii & Fabaceae & - & - & $\mathrm{Vu}$ & - & - & - & + \\
\hline Garcinia kola & Clusiaceae & - & - & $\mathrm{Vu}$ & - & + & - & + \\
\hline Guareacedrata & Meliaceae & HG & - & $\mathrm{Vu}$ & - & + & - & - \\
\hline Khayaivorensis & Meliaceae & - & - & $\mathrm{Vu}$ & + & + & - & + \\
\hline Leptoderrismiegei & Fabaceae & HG & GCi & - & - & + & - & + \\
\hline Lophiraalata & Ochnaceae & - & - & $\mathrm{Vu}$ & + & + & - & - \\
\hline Miliciaexcelsa & Moraceae & - & - & $\mathrm{Vu}$ & + & + & - & + \\
\hline Miliciaregia & Moraceae & HG & GCV & $\mathrm{Vu}$ & + & + & - & - \\
\hline Naucleadiderrichii & Rubiaceae & - & - & $\mathrm{Vu}$ & - & + & - & - \\
\hline Nesogordoniapapaverifera & Sterculiaceae & - & - & $\mathrm{Vu}$ & + & - & - & + \\
\hline Pericopsiselata & Fabaceae & - & - & En & - & - & - & + \\
\hline Tarrietiautilis & Sterculiaceae & HG & GCV & - & + & + & - & + \\
\hline Terminaliaivorensis & Combretaceae & - & - & $\mathrm{Vu}$ & - & + & - & + \\
\hline Tieghemellaheckelii & Sapotaceae & - & - & En & + & + & - & - \\
\hline Triplochitonscleroxylon & Sterculiaceae & - & - & $\mathrm{Vu}$ & - & + & - & - \\
\hline Turraeanthus africanus & Meliaceae & - & - & $\mathrm{Vu}$ & - & + & - & - \\
\hline
\end{tabular}

HG: espèces endémiques de la Haute Guinée ; GCW: espèces endémiques du bloc forestier Ouest africain, GCi: espèces endémiques de la Côte d'Ivoire; Vu: Vulnérable; EN: en danger; + présent; - absent; UICN : Union Internationale pour la Conservation de la Nature

Tableau 4: Densité, aire basale et biomasse aérienne des biotopes inventoriés du JBB Biotopes Densité (arbres/ha) Aire basale $\left(\mathrm{m}^{2} / \mathrm{ha}\right)$ Biomasse (t/ha)

$\begin{array}{cccc}\text { Arboretum de plus de } 100 \text { ans } & 430 & 10,92 & 266,7 \\ \text { Forêt } & 460 & 14,59 & 381,4 \\ \text { Jachère } & 88 & 1,04 & 21 \\ \text { Arboretum de } 20 \text { ans } & 460 & 13,16 & 300 \\ \text { total } & 362 & 39,72 & 472,5\end{array}$




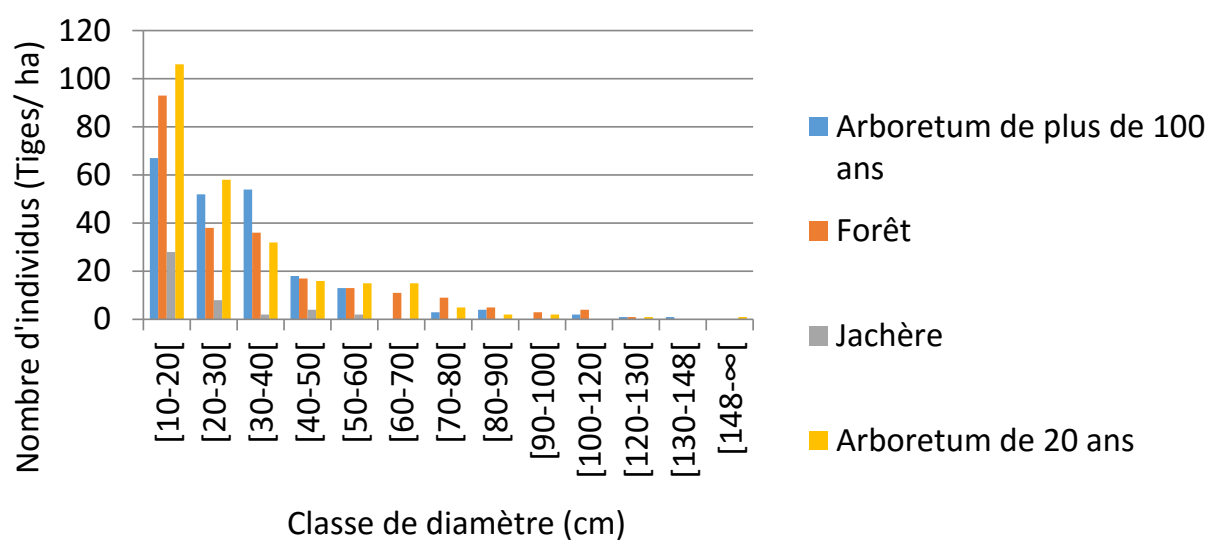

Figure 2: Histogrammes de distribution des tiges par classe de diamètre

La richesse floristique de toute la zone d'étude est estimée à 127 espèces arborées de $d b h \geq 10$ reparties entre 101 genres au sein de 39 familles. Cette valeur de la richesse floristique reste inférieure à celle obtenue par Séguéna et al. (2013) qui a signalé 419 taxons repartis entre 295 genres appartenant à 83 familles. Cet écart pourrait s'expliquer par le fait que la présente étude s'est intéressée seulement aux arbres matures ( $\mathrm{dbh} \geq 10$ ) contrairement à Séguéna et al. (2013) dont l'inventaire a pris en compte les espèces (lianes, herbes et arbres) de toutes morphologies. En effet, la diversité des espèces est influencée par les limites de diamètre, la prise en compte de diamètre plus petit permet de maximiser le nombre d'espèces étudiées dans un milieu (Vroh et al., 2010). Dans le JBB, la diversité des espèces serait aussi influencée par l'activité anthropique. En effet, l'introduction de nouvelles espèces lors des reboisements dans le JBB contribuerait à l'augmentation de la diversité. Depuis 1996, cette activité est beaucoup pratiquée par les gestionnaires du JBB. C'est ce qui se traduit par la valeur de l'indice de Shannon qui est plus élevée dans l'arboretum de 20 ans par rapport aux autres espaces. Le degré de ressemblance entre les parcelles confirme également cette situation car il n'excède pas les 50 p.c. Le plus élevé est établi entre l'arboretum de 20 ans et la forêt secondaire. Ces résultats démontrent que malgré la diversité de ces espaces, la flore présente est exclusive à chacun des espaces. Elle résulte majoritairement des choix faits des espèces lors des séances de reboisement.

Les espèces Cassia siamea et Terminalia mantaly sont prépondérantes dans l'arboretum de 20 ans; cela pourrait s'expliquer par le fait qu'il y ait eu une volonté de reboiser des espèces ornementales à croissance rapide. Dans cet espace, les espèces ont été plantées pour leur ombrage et ou pour des raisons paysagères. La prépondérance de Ceiba 
pentandra dans l'arboretum de plus de 100 ans est liée au fait que les actions de l'homme dans le passé a entrainé des ouvertures favorables à l'installation de cette espèce typique de formations secondaires (Alexandre, 1982). Par la suite, elle a été épargnée pour son ombrage et constitue aujourd'hui avec Milicia excelsa les arbres dominants de cet espace. La prépondérance de certaines espèces dans la jachère peut être liée à la volonté des cultivateurs de les épargner pour leur utilité ou pour leur ombrage dans les champs. L’absence de la majorité des espèces arborescentes dans la jachère, est liée au désherbage pendant la période culturale et à l'abondance des herbes pouvant ralentir la vitesse de reconstitution de la forêt pendant l'abandon (Khan, 1982).

Dans le jardin, 5 espèces sont endémiques des forêts de Haute Guinée (Porter et al., 2004), 17 sont inscrites sur la liste rouge de l'UICN (2015). Une autre espèce est endémique à la Côte d'Ivoire (Aké-Assi, 2001-2002). La présence de ces espèces est un signe de valeur de conservation pour la diversité biologique dans le JBB. Ce genre d'espèces mérite une attention particulière pour la conservation (Hawthorne, 1996 ; Jennings et al., 2003 ; Tchouto, 2004).

La forêt secondaire et l'arboretum de 20 ans sont les espaces les plus denses, cela est du au fait qu'il s'agit des espaces fermés par rapport aux autres qui sont ouverts. La structure diamétrique en forme de 'J inversé' obtenue dans chacun des espaces, excepté l'arboretum de plus de 100 ans, est typique aux forêts tropicales (Adou Yao, 2005). Cette courbe montre qu'en milieu naturel stable, l'effectif des individus d'un peuplement forestier diminue régulièrement lorsqu'on passe d'arbres de petits dbh aux plus gros arbres. Il s'agit d'un renouvèlement du peuplement arborescent des milieux tropicaux naturels. Mais la bonne capacité régénératrice des arbres des milieux reboisés du JBB est expliquée par les reboisements continus. Au niveau de l'arboretum de plus de 100 ans, le nettoyage régulier est la cause de l'interruption de la régénération des arbres d'où la forme de demi cloche. Pour l'ensemble du jardin, la densité de 362 arbres/ha diffère de l'intervalle défini par Pascal (2003), mais l'aire basale 39,72 $\mathrm{m}^{2} / \mathrm{ha}$ est comparable avec l'intervalle défini par cet auteur pour les forêts tropicales. Selon lui, la densité de tiges et l'aire basale sont respectivement compris entre 450-750 arbres/ha et $25-50 \mathrm{~m}^{2} / \mathrm{ha}$. Les valeurs obtenues pour ces deux paramètres montrent le caractère non naturel du jardin et elles peuvent être le résultat d'un certain nombre d'activités non contrôlées dans le jardin botanique pendant sa période d'abandon, en occurrence le prélèvement de plantes médicinales, de bois de chauffe et de construction. L'intensité de ces activités non contrôlées a eu un impact sévère sur la diversité, la diminution du nombre d'individus d'espèces et de la densité. 
La valeur de la biomasse est importante dans la forêt secondaire, elle a augmenté avec l'aire basale qui est liée à la grosseur des arbres (Dubé et al.,2006). Ceci pourrait être dû à une bonne diversité et surtout, à la présence d'espèces à forte capacité de stockage du carbone (Bakayoko et al., 2012). Ces espèces telles que Triplochiton scleroxylon, Ricinodendron heudelotii, Erythrina indica, Pentaclethra macrophylla ont aussi une forte densité spécifique, paramètre déterminant dans le calcul de la biomasse. La valeur de la biomasse est faible dans la jachère du fait de son caractère herbeux, donc moins riche en espèces arborescentes. La valeur de la biomasse comparée pour les deux arboretums montre que celle de l'arboretum de 20 ans est plus élevée. La valeur de la biomasse du JBB (472,5 t/ha) est élevée par rapport à celle obtenue au Centre National de Floristique par Kouamé (2013) qui est de 379,24 t/ha. Cette différence pourrait s’expliquer par le fait que le Centre National de Floristique est plus riche en espèces présentant un statut particulier pour la conservation, notamment la vulnérabilité. Ces espèces qui sont pour la plus part hors de leurs milieux naturels contiennent une faible biomasse et séquestrent moins de carbone. En effet, la capacité de séquestration de ces espèces serait d'autant plus forte qu'elles demeureraient dans leurs milieux naturels. Contrairement, les espèces du JBB ont une croissance rapide, donc elles ont une bonne capacité pour contenir la biomasse et séquestrer le carbone. La valeur moyenne de carbone séquestré par le JBB est de 236,2 $\pm 155,1$ tC/ha. Cette valeur qui tend vers celle définie

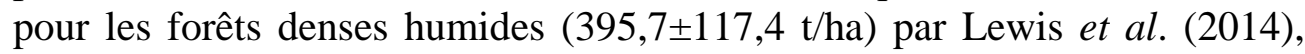
pourrait s'expliquer par le fait qu'en plus de quelques espèces à forte capacité de stockage de carbone, la prédominance de certaines espèces à croissance rapide (Cassia siamea, Acacia mangium) soit déterminante dans le stockage de carbone.

\section{Conclusion}

L’objectif de ce travail était de déterminer la diversité arborée et d'estimer le stock de carbone du Jardin Botanique de Bingerville (JBB) à travers quatre types de biotope : deux arboretums, une forêt et une jachère. $\mathrm{Au}$ terme de cette analyse, le JBB se présente comme un espace relativement diversifié. L’espace le plus reboisé (arboretum de 20 ans) est le plus riche en espèces que la forêt secondaire. L'on retient que le reboisement des espaces pour augmenter les superficies forestières peut servir à constituer de nouveaux réservoirs de carbone pour une période plus ou moins longue, selon la qualité et la croissance des espèces reboisées. La conservation de la diversité arborée du milieu urbain augmente la séquestration de carbone dans les arbres sur pied pour de longues durées, ce qui peut constituer un apport non négligeable dans le cadre du développement durable. 


\section{Remerciements}

Nous traduisons notre profonde gratitude et notre reconnaissance à l'endroit du Colonel GOUESSE Aïdara Lanciné, Directeur de cabinet du ministre des eaux et forêts pour avoir autorisé cette étude au sein du Jardin Botanique de Bingerville. Notre reconnaissance va également à l'endroit du Capitaine Coulibaly Sanga, Directeur du Jardin Botanique de Bingerville pour les informations qu'il a bien voulu nous communiquer sur le jardin. Nous remercions enfin les référés dont les contributions ont été très importantes pour l'amélioration de ce manuscrit.

\section{References:}

Adou Yao C. Y., Blom E. C., Denguéadhé K. T. S., Rompaey V R. S. A. R., N’Guessan K. E., Wittebolle G. \& Bongers F Diversité floristique et végétation dans le Sud du Parc National de Tai. Tropenbos - Côte d'Ivoire, Série 5, 91 p,2005.

Aké-Assi L. Impact de l'exploitation forestière et du développement agricole sur la conservation de la diversité biologique en Côte d'Ivoire. Le Flamboyant, 48 : 20-21, 1998.

Aké-Assi L. Flore de la Côte d'ivoire1, Catalogue Systématique, biogéographie et écologie, Conservation et jardin Botanique Genève, Suisse, 396p,2001.

Aké-Assi L. Flore de Côte d’Ivoire 2, catalogue systématique, biogéographie et écologie. Conservatoire et Jardin Botaniques, Genève, Switzerland, 401pp,2002.

Alexandre D. Y. Aspects de la régénération naturelle en forêt dense de Côte d’Ivoire. Caridollea 37 : 579-588, 1982.

Bakayoko A. Influence de la fragmentation forestière sur la composition floristique et structure végétale dans le Sud-ouest de la Côte d'Ivoire. Thèse Doctorat d’université, UFR Biosciences, Université Cocody Abidjan. 258 pp,2005.

Bakayoko O., Coulibaly B. et N'Guessan K. A.Carbonstorage in Nauclea didderichii and Triplochitonscleroxylon stand in Côte d'Ivoire. American Journal of Scientific Research 78: 133-142, 2012.

Brown S. et Lugo A.E. Aboveground biomass estimates for tropical moist forests of the Brazilian Amazon. Interciercia 17 (1): 8-18, 1992.

Brown S. Estimating biomass and biomass changes of tropical forest: A primer.FAO Forestry Paper 134, Rome, Italy, 1997.

Chave J, Andalo C, Brown S, Cairns MA, Chambers JQ, Eamus D, Fölster H, Fromard F, Higuchi N, Kira T, Lescure J-P, Nelson BW, Ogawa H, Puig H, Riéra B, Yamakura T. Tree allometry and improved estimation of carbon stocks and balance in tropical forests. Oecologia, 145:87-99, 2005. 
Cottam G. and Curtis, J.T. The use of distance measures in phytosociological sampling. Ecology 37 : 451-460, 1956.

Doumbia M. Diversité, caractéristiques biomorphologiques des espèces à statuts particuliers de l'arboretum du Centre National de Floristique (CNF) de l’Université Félix Houphouët Boigny. Mémoire de Master UFR Biosciences, Université FHB, Abidjan, 55 p, 2014.

Dubé A.D. S.L. et G. Sénécal., Penser le renouvellement des politiques de conservation de la forêt urbaine à l'ère du réchauffement climatique. Institut National de la Recherche Scientifique-Urbanisation, culture et société, 51 p,2006.

Edlin E. Le climat in : Avenard J.M ; Edlin E. ; Girard G.; Sircoulon J.; Touchebeuf P.; Guillaumet J.L.; Adjanohoun E; Perraud A.; 1971. Le milieu naturel de la Côte d’Ivoire. ORSTOM, Paris, France: pp 73-108,1971.

FAO, 2011. Situation des forêts du monde, Rome, 586 pp.

Hawthorne W. D. Guide de terrain pour les arbres des forêts denses de la Côte d'Ivoire et des pays limitrophes. Avec clés végétatifs sur plus de 650 espèces d'arbres, à partir de $5 \mathrm{~cm}$ de diamètre. Université Agronomique de Wageningen (Pays Bas), 279 p, 1996.

Jennings S, Nussbaum R, Judd J, Evans T. The High Conservation Value Forest Toolkit Edition 1, Pro Forest, 27, 2003.

Khan F. La reconstitution de la forêt tropicale après une culture traditionnelle, (Sud oust de la côte d'Ivoire). Paris: ORSTOM. 150 pp,1982.

Kouamé A.S. Diversité végétale et estimation de la biomasse dans l'arboretum du centre national de floristique (Abidjan, Côte d'Ivoire). Mémoire de DEA UFR Biosciences, Université FHB, Abidjan, 2013.

Kouassi M. O. Inventaire floristique et régénération des espèces arbustives, lianescentes et herbacées de la parcelle Henry Konan Bédié du JBB (Côte d'Ivoire). Mémoire de Maîtrise UFR des Sciences de la Nature Université d’Abobo-Adjamé,2010.

Kpangui K.B. Apport des Systèmes d’Informations Géographiques à l'étude de la diversité spécifique de l'arboretum du Centre National de Floristique (Côte d'Ivoire). Mémoire de DEA UFR Biosciences Université de Cocody, Abidjan, 75 p, 2009.

Lewis SL, Sonke' B, Sunderland T, Begne SK, Lopez-Gonzalez G, van der Heijden GMF, Phillips OL, Affum-Baffoe K, Baker TR, Banin L, Bastin FJ, Beeckman H, Boeckx P, Bogaert J, Cannière DC, Chezeaux E, Clark CJ, Collins M, Djagbletey G, Djuikouo MNK, Droissart V, Doucet JL, Ewango CEN, Fauset S, Feldpausch TR, Foli EG, Gillet JF, Hamilton AC, Harris DJ, Hart TB, de Haulleville T, Hladik A, Hufkens K, Huygens D, Jeanmart P, Jeffery KJ, Kearsley E, Leal ME, Lloyd J, Lovett JC, Makana J-R, Malhi Y, Marshall AR, Ojo L, Peh KS-H, Pickavance G, Poulsen JR, Reitsma JM, Sheil D, Simo M, Steppe K, Taedoumg HE, Talbot J, Taplin JRD, Taylor D, 
Thomas SC, Toirambe B, Verbeeck H, Vleminckx J, White LJT, Willcock S, Woell H, Zemagho L. Above-grounds biomasse and structure of 260 African tropical forets. Philosophical transactions:1-14,2014.

Mangenot G. Etude sur les forêts des plaines et des plateaux de la Côte d’Ivoire. Etude Eburnéenne. IFAN 61 p,1955.

N’Guessan K. E., Vroh B. T. A., Ouattara D., Tibre M.S., Adou Y. C. Y. Le Parc National Du Banco, Une Forêt Urbaine Au Service Du Bien-être Des Populations d'Abidjan (Côte d'Ivoire). XIVe Congrès forestier mondial Durban, Afrique du Sud, 2015.

Nowak D.J, Crane, D.E., Stevens, J. C Air Pollution Removal by Urban Trees and Shrubs in the United States. Urbana Forester \& Urbana Genin, 4: 115-123, 2006.

Nusbaumer L, Gautier L. et Chatelain C., Structure et composition de la Forêt Classée du Scio (Côte d'Ivoire) Etude descriptive et comparative. Candollea60 (2): 393-443,2005.

Pascal J-P. Notions sur les structures et dynamique des forêts tropicales humides. Rev. For. Fr. LV - Numéro spécial: 118-130, 2003.

Piélou E. C., Species diversity and pattern diversity in the study of ecological succession. Journal of theoretical biology, 10: 370 - 383. 1966

Porter L., Bingers F., Kotare F. N'. \& Hawthorne W. D., Biodiversity of West African Forests: An Ecological Atlas of Woody Plant Species. CABI Publishing, Nederland, Pays-Bas, 521 p, 2004.

Puig H. La forêt tropicale humide. Editions Belin, Paris, 448p, 2001.

Raunkiaer C. The life forms of plants and statistical geography. Claredon, Oxford 632 p, 1934.

Séguéna F., Soro K., Soro D., N’Guessan K. Savoir faire des populations locales des taxons du Jardin Botanique de Bingerville, Côte d'Ivoire. Journal of Applied biosciences 68: 5374-5393, 2013.

Shannon C.E. A mathematical theory of communications. Bell. System Technical Journal 27: 379-423, 1948.

Sørensen T.A method of establishing groups of amplitude in sociology based on similarity of content, and its application to analysis of vegetation on Danish commons Biologisfter5: 1-34,1948.

Tchouto M.G.P. Plant diversity in central African rain forest. Implications for biodiversity conservation in Cameroon. $\mathrm{PhD}$ thesis, Wageningen University, Wageningen, The Netherlands. 208p,2004.

UICN. IUCN Read List of Treatement Species .www iucnreadlist. org. 2015. Vroh Bi T. A., Adou Yao C.Y., Kouamé D., N'da D.H., N’Guessan K.E. Diversités Floristique et structurale sur le site d'une réserve naturelle volontaire à Azaguié, Sud-est de la Côte d'Ivoire. European Journal of Scientific Research (45): 1450 - 216, 2010 
Vroh Bi T. A., Marie S. T., Kouakou E. N., . Diversité végétale urbaine et estimation du stock de carbone: cas de la commune du Plateau Abidjan, Côte d’Ivoire. Afrique Sciences 10(3) : 329-340. 2014

Weldenson D. Evaluation de la biomasse et des stocks de carbone sur des placettes forestières en forêts tropicales humides de la Guadeloupe, 22 $\mathrm{p}, 2010$. 